\title{
Dynorphin Counteracts Orexin in the Paraventricular Nucleus of the Thalamus: Cellular and Behavioral Evidence
}

\author{
Alessandra Matzeu*,', Marsida Kallupi', Olivier George', Paul Schweitzer' and Rémi Martin-Fardon' \\ 'Department of Neuroscience, The Scripps Research Institute, La Jolla, CA, USA
}

\begin{abstract}
The orexin (Orx) system plays a critical role in drug addiction and reward-related behaviors. The dynorphin (Dyn) system promotes depressive-like behavior and plays a key role in the aversive effects of stress. Orx and Dyn are co-released and have opposing functions in reward and motivation in the ventral tegmental area (VTA). Previous studies suggested that OrxA transmission in the posterior paraventricular nucleus of the thalamus (PPVT) participates in cocaine-seeking behavior. This study determined whether Orx and Dyn interact in the PPVT. Using the brain slice preparation for cellular recordings, superfusion of DynA onto pPVT neurons decreased the frequency of spontaneous and miniature excitatory postsynaptic currents (s/mEPSCs). OrxA increased the frequency of sEPSCs but had no effect on mEPSCs, suggesting a network-driven effect of OrxA. The amplitudes of s/mEPSCs were unaffected by the peptides, indicating a presynaptic action on glutamate release. Augmentation of OrxA-induced glutamate release was reversed by DynA. Utilizing a behavioral approach, separate groups of male Wistar rats were trained to self-administer cocaine or sweetened condensed milk (SCM). After extinction, rats received intra-pPVT administration of OrxA \pm DynA \pm the $\kappa$-opioid receptor antagonist nor-binaltorphimine (NorBNI) under extinction conditions. OrxA reinstated cocaine- and SCM-seeking behavior, with a greater effect in cocaine animals. DynA blocked OrxA-induced cocaine seeking but not SCM seeking. NorBNI did not induce or potentiate cocaine-seeking behavior induced by OrxA but reversed DynA effect. This indicates that the $\kappa$-opioid system in the PPVT counteracts OrxA-induced cocaine seeking, suggesting a novel therapeutic target to prevent cocaine relapse.

Neuropsychopharmacology (2018) 43, I0 I0-1020; doi:10.1038/npp.2017.250; published online 22 November 2017
\end{abstract}

\section{INTRODUCTION}

Orexin A (OrxA or hypocretin-1) and orexin B (OrxB or hypocretin-2) are hypothalamic neuropeptides (de Lecea et al, 1998; Sakurai et al, 1998) that regulate feeding, energy metabolism, and arousal (James et al, 2016; Li et al, 2016). Two Orx receptors (OxRs) have been identified, OxR1 and OxR2 (Ammoun et al, 2003; Sakurai et al, 1998). OrxA binds OxR1 and OxR2 with similar affinity, whereas OrxB binds OxR2 with higher affinity (Ammoun et al, 2003; Sakurai et al, 1998). Orexin-expressing neurons are exclusively found in the hypothalamus (de Lecea et al, 1998; Sakurai et al, 1998) and project throughout the brain (Peyron et al, 1998), playing a key role in modulating reward function, particularly drug-directed behavior (Harris et al, 2005). Orexin has been reported to enhance the incentive motivational effects of stimuli that are conditioned to drug availability, increase the motivation to seek drugs of abuse, and enhance the reinforcing actions of drugs of abuse (for review, see Matzeu et al, 2014).

* Correspondence: Dr A Matzeu, Department of Neuroscience, The Scripps Research Institute, 10550 North Torrey Pines Road, SP30-2003, La Jolla, CA 92037, USA, Tel: + I 858784 7339, Fax: + I 858784 7243, E-mail: amatzeu@scripps.edu

Received 3 March 2017; revised 13 October 2017; accepted 15 October 2017; accepted article preview online 20 October 2017
The dynorphin/ $\kappa$-opioid receptor (Dyn/KOR) system is widely distributed in the brain (Watson et al, 1982) and implicated in numerous physiological and pathophysiological processes that are related to mood and motivation (Schwarzer, 2009; Wee and Koob, 2010). The Dyn/KOR system has been suggested to be a putative therapeutic target for the treatment of various neuropsychiatric disorders (Schwarzer, 2009). Dynorphin (Dyn) and $\alpha$-neo-endorphin are endogenous opioid peptides that are generated from the precursor prodynorphin by posttranslational proteolytic cleavage, and these peptides bind KORs (Schwarzer, 2009). Two forms of Dyn (DynA and DynB) exist, and both forms (especially DynA) have a marked preference for KORs (Chavkin et al, 1982). In contrast to Orx, which promotes arousal and is implicated in the rewarding effects of food, sexual behavior, and drugs of abuse (Tsujino and Sakurai, 2013), Dyn promotes depressive-like behavior and plays a key role in mediating the aversive effects of stress (Bruchas et al, 2010). The activation of KORs can attenuate the rewarding effects of drugs of abuse (Wee and Koob, 2010).

Despite their opposing effects on motivation, Orx neurons contain Dyn (Chou et al, 2001). Evidence suggests that these peptides act in concert. For example, both Orx and Dyn are co-released during electrical stimulation of the hypothalamus ( $\mathrm{Li}$ and van den Pol, 2006) and play opposing roles in cocaine self-administration, brain stimulation reward, and impulsivity (Muschamp et al, 2014). At the cellular level, 
OrxA increases and DynA decreases the firing rate of ventral tegmental area (VTA) neurons (Muschamp et al, 2014; Baimel et al, 2017). The co-application of these peptides resulted in no net changes in neuronal firing, suggesting that OrxA and DynA exert balanced and opposing effects on VTA neurons (Muschamp et al, 2014), perhaps by modulating different circuits within the VTA (Baimel et al, 2017).

The paraventricular nucleus of the hypothalamus (PVT) plays an important role in functions that are related to arousal, attention, and awareness (Van der Werf et al, 2002). Data from our laboratory demonstrated a role for the posterior PVT (pPVT) and particularly Orx transmission in the pPVT in cocaine-seeking behavior (Matzeu et al, 2017; Matzeu et al, 2015, 2016). Few $\gamma$-aminobutyric acid-ergic neurons are found in local PVT circuitry, indicating that the synaptic network is mostly glutamatergic in this midline nucleus (Arcelli et al, 1997). Neurons in the PVT receive one of the densest Orx projections in the brain (Kirouac et al, 2005; Peyron et al, 1998), and KOR immunoreactivity is high in these neurons (Mansour et al, 1996). In the PVT, Orx and Dyn modulate ionic conductance and neuronal activity in opposite directions (Chen et al, 2015; Kolaj et al, 2014), but the regulation of excitatory synaptic transmission by Orx is poorly understood, and the effect of Dyn is unknown. To determine whether functional interactions exist between Orx and Dyn in the pPVT, whole-cell recordings were performed, and drug-seeking behavior was evaluated.

\section{MATERIALS AND METHODS}

\section{Rats}

Male Wistar rats $(n=134$; Charles River, Wilmington, MA), weighing 200-225 g (2 months old) upon arrival, were housed two per cage in a temperature- and humidity-controlled vivarium on a reverse $12 \mathrm{~h} / 12 \mathrm{~h}$ light/dark cycle with ad libitum access to food and water. All of the procedures were conducted in strict adherence to the National Institutes of Health Guide for the Care and Use of Laboratory Animals and were approved by the Institutional Animal Care and Use Committee of The Scripps Research Institute.

\section{Drugs}

OrxA, DynA (American Peptide, Sunnyvale, CA), and norbinaltorphimine dihydrochloride (NorBNI; Sigma-Aldrich, St Louis, MO) were diluted in $0.9 \%$ sodium chloride (Hospira, Lake Forest, IL) and injected in a volume of $0.5 \mu$ l. Cocaine (National Institute on Drug Abuse, Bethesda, MD) was dissolved in $0.9 \%$ sodium chloride (Hospira, Lake Forest, IL) and administered at a dose of $0.25 \mathrm{mg} / 0.1 \mathrm{ml}$. Sweetened condensed milk (SCM; Nestlé, Solon, OH) was diluted $2: 1(\mathrm{v} / \mathrm{v})$ in water and delivered in a volume of $0.1 \mathrm{ml}$. For electrophysiology, OrxA, DynA, and NorBNI were dissolved in the superfusate, bicuculline, or picrotoxin (Sigma, St Louis, MO) and TCSOX229 were superfused in 0.05-0.1\% dimethylsulfoxide.

\section{Electrophysiology}

pPVT slices were prepared from naive rats (20 rats total, 2-4 rats per experiment) that were age matched to the rats that were used in the behavioral experiments. The rats were deeply anesthetized with isoflurane, and brains were rapidly removed and placed in oxygenated $\left(95 \% \mathrm{O}_{2}, 5 \% \mathrm{CO}_{2}\right)$ icecold cutting solution that contained $206 \mathrm{mM}$ sucrose, $2.5 \mathrm{mM} \mathrm{KCl}, 1.2 \mathrm{mM} \mathrm{NaH} \mathrm{PO}_{4}, 7 \mathrm{mM} \mathrm{MgCl}_{2}, 0.5 \mathrm{mM}$ $\mathrm{CaCl}_{2}, 26 \mathrm{mM} \mathrm{NaHCO}, 5 \mathrm{mM}$ glucose, and $5 \mathrm{mM}$ HEPES. Transverse slices $(300 \mu \mathrm{m}$ thick) were cut on a Vibratome (Leica VT1000S, Leica Microsystems, Buffalo Grove, IL) and transferred to oxygenated artificial cerebrospinal fluid (aCSF) that contained $130 \mathrm{mM} \mathrm{NaCl}, 2.5 \mathrm{mM} \mathrm{KCl}, 1.2 \mathrm{mM}$ $\mathrm{NaH}_{2} \mathrm{PO}_{4}, 2.0 \mathrm{mM} \mathrm{MgSO} \cdot 7 \mathrm{H}_{2} \mathrm{O}, 2.0 \mathrm{mM} \mathrm{CaCl}, 26 \mathrm{mM}$ $\mathrm{NaHCO}_{3}$, and $10 \mathrm{mM}$ glucose. The slices were first incubated for $30 \mathrm{~min}$ at $35^{\circ} \mathrm{C}$ and then kept at room temperature for the remainder of the experiment. Individual slices were transferred to a recording chamber that was mounted on the stage of an upright microscope (Olympus BX50WI). The slices were continuously perfused with oxygenated aCSF at a rate of $2-3 \mathrm{ml} / \mathrm{min}$. Neurons were visualized with a $60 \times$ water immersion objective (Olympus), infrared differential interference contrast optics, and a charge-coupled device camera (EXi Blue from QImaging, Surrey, Canada). Wholecell recordings were performed using a Multiclamp 700B amplifier (10 kHz sampling rate, $10 \mathrm{kHz}$ low-pass filter) and Digidata 1440A and pClamp 10 software (Molecular Devices, Sunnyvale, CA). Patch pipettes (4-7 $M^{\prime} \Omega$ ) were pulled from borosilicate glass (Warner Instruments, Hamden, CT) and filled with $70 \mathrm{mM} \mathrm{KMeSO}_{4}, 55 \mathrm{mM} \mathrm{KCl}, 10 \mathrm{mM} \mathrm{NaCl}, 2 \mathrm{mM}$ $\mathrm{MgCl}_{2}, 10 \mathrm{mM}$ HEPES, $2 \mathrm{mM} \mathrm{Na-ATP}$, and $0.2 \mathrm{mM} \mathrm{Na}-$ GTP. Liquid junction potential corrections were performed offline. Pharmacologically isolated spontaneous excitatory postsynaptic currents (sEPSCs) were recorded in the presence of picrotoxin $(20 \mu \mathrm{M})$ or bicuculline $(30 \mu \mathrm{M})$. Experiments with a series resistance of $>15 \mathrm{M} \Omega$ or $>20 \%$ change in series resistance were excluded from the final data set. The frequency, amplitude, and kinetics of spontaneous/ miniature EPSCs (s/mEPSCs) were analyzed using a semiautomated threshold-based mini detection software (MiniAnalysis, Synaptosoft, Fort Lee, NJ) and visually confirmed. To obtain a high signal-to-noise ratio and accurately determine the $\mathrm{s} / \mathrm{mEPSC}$ amplitude, only events $>10 \mathrm{pA}$ were accepted for analysis. Averages of s/mIPSC characteristics were based on a minimum time interval of $3 \mathrm{~min}$ and a minimum of 50 events.

\section{Behavior}

Self-administration and extinction training. Cocaine. Rats $(n=64)$ were surgically prepared with jugular catheters 7-10 days before cocaine self-administration training in daily $6 \mathrm{~h}$ (long access) sessions (Figure 1a). Every session was initiated by the extension of two retractable levers into the operant chamber $(29 \mathrm{~cm} \times 24 \mathrm{~cm} \times 19.5 \mathrm{~cm}$, Med Associates, St Albans, VT). Responses on the right active lever were reinforced on a fixed-ratio 1 (FR1) schedule by intravenous (IV) cocaine $(0.25 \mathrm{mg} / 0.1 \mathrm{ml} /$ infusion $)$ that was infused over $4 \mathrm{~s}$ followed by a $20 \mathrm{~s}$ timeout (TO20) period that was signaled by illumination of a cue light above the active lever. Responses on the left inactive lever were recorded but had no scheduled consequences. Sweetened condensed milk. SCM $(n=50)$ self-administration was established in daily $30 \mathrm{~min}$ sessions on an FR1 TO20 schedule of reinforcement. Sessions were initiated by the extension of both levers into 
a

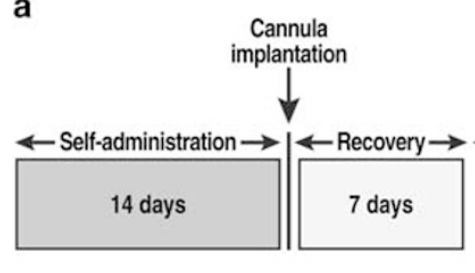

b

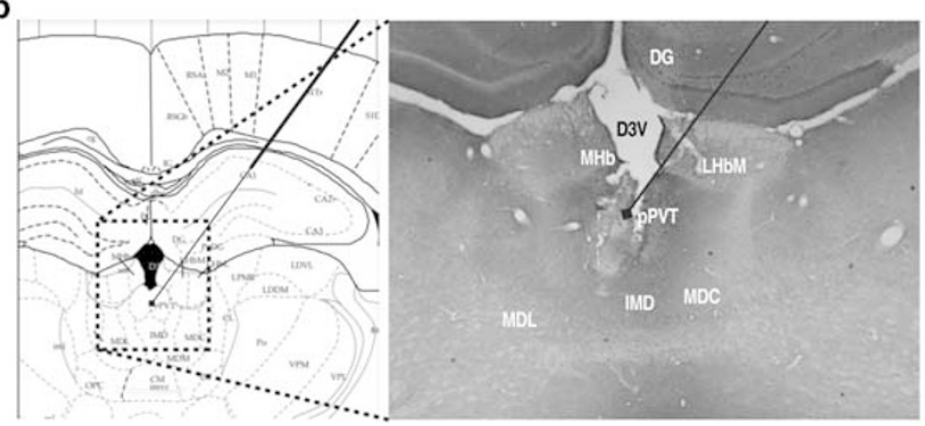

c

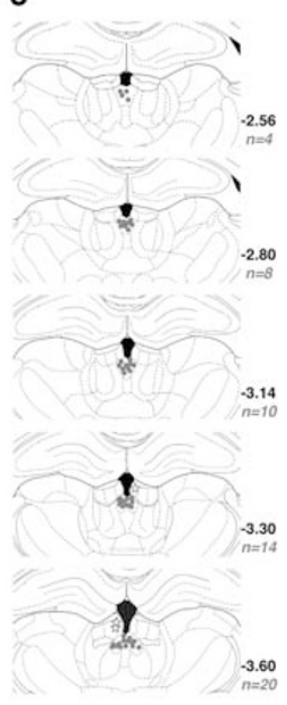

d

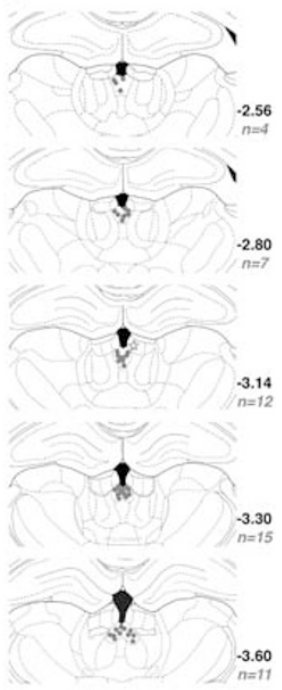

Figure I (a) Behavioral procedure. (b) Schematic representation of injector placements in the pPVT (Paxinos and Watson, 1997), with photo representation of the injection track ( $\times 2.5$ magnification). (c) Injection sites for cocaine and (d) SCM animals. These schematics represent coronal sections of rat brains encompassing the midpoint of accurate injection sites (gray dots) in the anterior-posterior plane. All drawings are based on the atlas of Paxinos and Watson (1997). The $n$ values on the graphs represent the number of animals/coronal sections. Gray stars represent missed injections (three for cocaine and one for SCM).

the operant chamber, and responses on the right active lever resulted in the delivery of SCM $(0.1 \mathrm{ml})$ into a drinking receptacle. Responses on the left inactive lever were recorded but had no scheduled consequences. Cannulation. After 14 self-administration sessions, both groups were implanted with a guide cannula (23 gauge, $15 \mathrm{~mm}$, Plastics One, Roanoke, VA) that was aimed at the pPVT (anterior/ posterior, $-3.3 \mathrm{~mm}$; medial/lateral, $\pm 2.72 \mathrm{~mm}$ from bregma; dorsal/ventral, $-2.96 \mathrm{~mm}$ from dura, at a $25^{\circ}$ angle; Paxinos and Watson, 1997) and positioned $3.5 \mathrm{~mm}$ above the target injection point (Figure $1 \mathrm{~b}-\mathrm{d}$ ). After 7 days of recovery, the animals resumed self-administration training for an additional 7 days. Extinction. The day following the completion of self-administration training, the rats were placed under extinction (EXT) conditions (extinction criterion: $\leqslant 10$ active lever presses over the last three sessions). All of the extinction sessions lasted $2 \mathrm{~h}$ and were identical to the selfadministration sessions but without reward (cocaine or SCM) availability.

Intra-pPVT microinjection. On the last day of extinction training, each rat received a sham injection for habituation to the microinjection (Figure 1a). After $24 \mathrm{~h}$, each animal received an intra-pPVT microinjection of $0.5 \mu \mathrm{g}$ OrxA (the minimal dose that has been shown to induce cocaine-seeking behavior; Matzeu et al, 2016) \pm Dyn-A $(0,1,2$, or $4 \mu \mathrm{g})$ or DynA alone $(4 \mu \mathrm{g})$ using a micro-infusion pump (Harvard 22 Syringe Pump, Holliston, MA). The injectors extended $3.5 \mathrm{~mm}$ beyond the guide cannula. Injections were performed at a flow rate of $0.5 \mu \mathrm{l} / \mathrm{min}$ over $1 \mathrm{~min}$. The injector was kept in place for an additional minute to allow diffusion. Following the injections, the rats were returned to their home cage for $2 \mathrm{~min}$ and then transferred to the operant chambers and tested under extinction conditions for $2 \mathrm{~h}$. A separate cohort of rats was prepared to test effects of KOR blockade on OrxA-induced reinstatement. Briefly, rats were trained to self-administer cocaine $(\operatorname{Lg} A)$ as described above and then received an intra-pPVT microinjection of $4 \mu \mathrm{g}$ NorBNI 1 day before the reinstatement test. The dose of NorBNI and injection time were based on previous studies (Whitfield et al, 2015). After $24 \mathrm{~h}$, the rats then received an intra-pPVT injection of OrxA $(0.5 \mu \mathrm{g})$ or OrxA+DynA $(0.5$ and $4 \mu \mathrm{g}$, respectively) and tested again for cocaine seeking. The injection procedure and the reinstatement test for this extra group of animals were identical to the procedures described above.

\section{Histology}

Upon completion of the reinstatement test, the rats were killed by $\mathrm{CO}_{2}$ inhalation. The brains were then collected and snap frozen. The brains were then sliced into $40 \mu \mathrm{m}$ coronal sections, and injector placements in the pPVT were verified (Figure 1b-d).

\section{Statistical Analysis}

The electrophysiology data $($ mean \pm SEM) were analyzed using paired $t$-tests, one-way analysis of variance (ANOVA), or one-way repeated-measures ANOVA followed by the Tukey's post hoc test when appropriate. For the behavioral data, cocaine and SCM intake during self-administration was analyzed using one-way repeated-measures ANOVA. The number of days to meet the extinction criterion for cocaine and SCM self-administration was compared using unpaired $t$-tests. Differences between extinction, sham, and reinstatement after the vehicle injection were analyzed using two-way repeated-measures ANOVA. Reinstatement following the OrxA, DynA, and OrxA+DynA injections was first analyzed by two-way and then individual one-way ANOVAs. 
Reinstatement following the NorBNI, NorBNI+OrxA, and NorBNI+OrxA+DynA injections was analyzed by one-way ANOVAs. Tukey's post hoc tests were performed to confirm significance.

\section{RESULTS}

Eight animals were lost in the cocaine groups (two because of health complications, three because of catheter failure, and three because of injection misplacement (one from the VEH subgroup, one from the OrxA subgroup that did not reinstate, and one from the NorBNI+OrxA+DynA subgroup that did not reinstate as well)). One rat was lost in the SCM group because of injection misplacement (from the VEH subgroup), thus reducing the number of animals to 125 ( $n=20$ naive rats for electrophysiology, $n=44$ rats for cocaine first cohort, $n=12$ rats for cocaine second cohort, and $n=49$ rats for SCM). It is important to note that even though no anatomical controls were prepared, the behavioral data obtained for misplaced injection $(<4 \%)$ suggest that missed injection of OrxA in the pPVT did not produce any behavioral effects, confirming that the reinstating effect of OrxA was pPVT specific.

\section{DynA Decreased and OrxA Increased Glutamatergic Transmission in Naive pPVT Neurons}

The modulation of glutamatergic transmission by OrxA and DynA was assessed by studying s/mEPSCs in pPVT neurons (Figure 2a). The resting membrane potential of the neuronal sample was $-65 \pm 0.9 \mathrm{mV}$, the input resistance was $234 \pm 12 \mathrm{M} \Omega$, and the holding potential was $-68 \pm 0.5 \mathrm{mV}$ $(n=45)$. We assessed the effects of the peptides on sEPSCs to determine their influence on action potential-dependent synaptic transmission. DynA $(0.2 \mu \mathrm{M})$ significantly decreased the frequency of sEPSCs in pPVT neurons by $40 \pm 6 \%$ (from $1.57 \pm 0.27 \mathrm{~Hz} \quad$ (control/baseline) to $0.98 \pm 0.22 \mathrm{~Hz} ; n=10$ cells $/ 4$ rats; paired $t$-test, $t_{9}=3.9$, $p<0.01$; Figure $2 \mathrm{~b}-\mathrm{d}$ ). The amplitude (baseline $42 \pm 4 \mathrm{pA}$ ), rise time $(1.8 \pm 0.1 \mathrm{~ms})$, and decay time $(3.8 \pm 0.5 \mathrm{~ms})$ of sEPSCs were not significantly affected by DynA and remained at $94 \pm 6 \%$ (Figure $2 \mathrm{~d}$ ), $97 \pm 3 \%$, and $100 \pm 8 \%$ of control, respectively $(n=10)$. In the presence of the KOR antagonist NorBNI $(0.2 \mu \mathrm{M})$, DynA had no significant effect on the frequency of sEPSCs that remained at $97 \pm 4 \%$ of control $(n=5$ cells $/ 2$ rats; $p>0.05)$. The amplitude, rise time, and decay time of sEPSCs were also unaffected (data not shown). In contrast, OrxA $(1 \mu \mathrm{M})$ significantly increased the frequency of sEPSCs in pPVT neurons by $38 \pm 6 \%$ (from $1.49 \pm 0.24$ to $1.96 \pm 0.28 \mathrm{~Hz} ; n=10$ cells $/ 4$ rats; paired $t$-test, $t_{9}=8.7, p<0.001$; Figure $2 \mathrm{f}$ and $\mathrm{g}$ ). The amplitude (baseline $48 \pm 4 \mathrm{pA})$, rise time $(1.9 \pm 0.1 \mathrm{~ms})$, and decay time $(3.6 \pm 0.5 \mathrm{~ms})$ of sEPSCs were not significantly affected by OrxA, remaining at $101 \pm 5 \%$ (Figure $2 \mathrm{~g}$ ), $97 \pm 2 \%$, and $96 \pm 6 \%$ of control, respectively $(n=10)$. In the presence of the Orx receptor antagonist TCSOX229, OrxA had no significant effect on the frequency $(95 \pm 5 \%$ of control, $n=5$ cells $/ 2$ rats; $p>0.05)$, amplitude, rise time, or decay time of sEPSCs.

We also recorded synaptic activity in the presence of $0.5 \mu \mathrm{M}$ tetrodotoxin to study the effect of DynA and OrxA on action potential-independent glutamate release (mEPSCs). With action potentials blocked, DynA $(0.2 \mu \mathrm{M})$ significantly decreased the frequency of mEPSCs by $40 \pm 4 \%$ (from $1.08 \pm 0.21$ to $0.63 \pm 0.13 \mathrm{~Hz} ; n=6$ cells $/ 3$ rats; paired $t$-test, $t_{5}=9.145, p<0.01$; Figure $2 \mathrm{e}$ ), a value similar to that obtained with sEPSCs. DynA did not significantly alter mEPSC amplitude (baseline $35 \pm 2 \mathrm{pA}$ ) that remained at $99 \pm 3 \%$ of control (Figure 2e), rise time or decay time. Superfusion of OrxA $(1 \mu \mathrm{M})$, however, had no significant effect on the frequency of mEPSC (baseline $1.14 \pm 0.14 \mathrm{~Hz}$ ) that remained at $98 \pm 5 \%$ of control in presence of the peptide $(n=6$ cells/ 3 rats; $p>0.05$; Figure $2 \mathrm{~h}$ ), in sharp contrast to the effect on sEPSC frequency. OrxA had no significant effect on mEPSC amplitude (baseline $32 \pm 3 \mathrm{pA}$ ) that remained at $104 \pm 10 \%$ of control (Figure $2 \mathrm{~h}$ ), rise time, and/or decay time.

\section{DynA Reversed the Effects of OrxA on Glutamatergic Transmission in Naive pPVT Neurons}

The interaction between OrxA and DynA was studied by first applying OrxA alone and then combined with DynA in the continued presence of OrxA. In this neuronal sample, the exposure of pPVT neurons to OrxA $(1 \mu \mathrm{M})$ significantly increased the frequency of sEPSCs from $1.58 \pm 0.23$ to $2.04 \pm 0.27 \mathrm{~Hz}$ (Tukey's post hoc test, $p<0.01$; one-way repeated-measures ANOVA, $\mathrm{F}_{2,7}=68.1, p<0.01$; Figure $3 \mathrm{a}$ and $b)$. The addition of DynA $(200 \mathrm{nM})$ completely reversed the effect of OrxA to control levels (sEPSC frequency: $96 \pm 8 \%$, control $v s$ OrxA+DynA, $p>0.05 ; n=8$ cells from 3 rats; Figure 3$)$. The addition of DynA in the presence of OrxA did not significantly affect the sEPSC amplitude (baseline $46 \pm 3 \mathrm{pA}$ ) that remained at $98 \pm 2 \%$ of OrxA levels, rise time $(103 \pm 3 \%$ of OrxA), or decay time $(95 \pm 5 \%$ of OrxA). Tukey's post hoc test revealed that the co-application of OrxA+DynA significantly decreased sEPSCs compared with OrxA alone $(p<0.01)$ and significantly increased sEPSCs compared with DynA alone $(p<0.01)$, suggesting that the combination of OrxA+DynA reversed the effect that was observed with each peptide individually (Figure $3 \mathrm{c}$ ).

\section{Cocaine and SCM Self-Administration Training and Extinction}

In daily $6 \mathrm{~h}$ cocaine self-administration sessions, the amount $(\mathrm{mg} / \mathrm{kg})$ of cocaine that was self-administered gradually increased (repeated-measures ANOVA, $\mathrm{F}_{43,860}=25.1$, $p<0.001)$ from day $1 \quad(15.2 \pm 2.3 \mathrm{mg} / \mathrm{kg})$ to day 7 $(51.6 \pm 4.9 \mathrm{mg} / \mathrm{kg})$ and then remained unchanged until the end of self-administration training (Figure $4 \mathrm{a}$ ). In daily 30 min sessions of SCM self-administration, the amount (g/ $\mathrm{kg}$ ) of SCM that was self-administered gradually increased (repeated-measures ANOVA, $\mathrm{F}_{48,960}=14.3, p<0.001$ ) from day $1(1.4 \pm 0.2 \mathrm{~g} / \mathrm{kg})$ to day $7(7.6 \pm 0.5 \mathrm{~g} / \mathrm{kg})$ and then remained unchanged until the end of self-administration training (Figure 4b). By the end of extinction training $(19.2 \pm 0.3$ days for cocaine and $18.8 \pm 0.2$ days for SCM; unpaired $t$-test, $t_{91}=1.01, p>0.05$ ), the rats reached a 3 -day average $( \pm$ SEM) number of responses of $8.4 \pm 1.1$ for cocaine and $8.2 \pm 1.5$ for SCM. No differences were observed between the number of responses at the end of extinction, or following sham or vehicle injection in the cocaine group $v s$ 


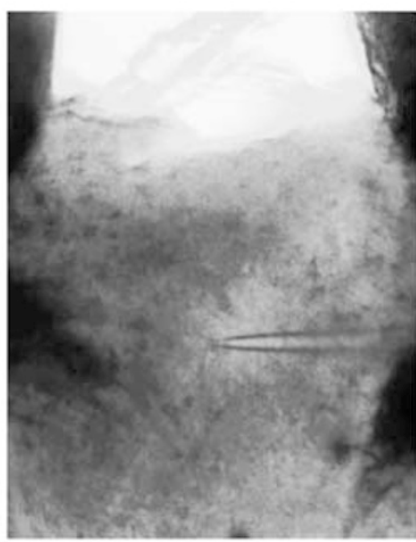

C

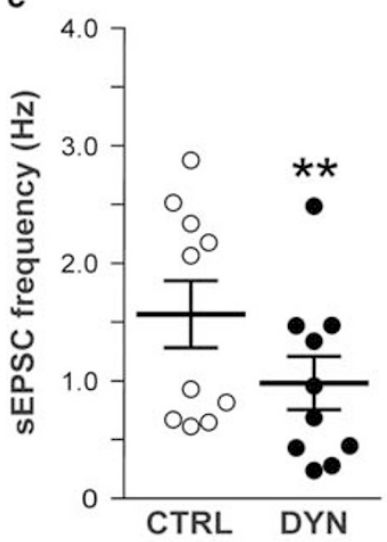

f

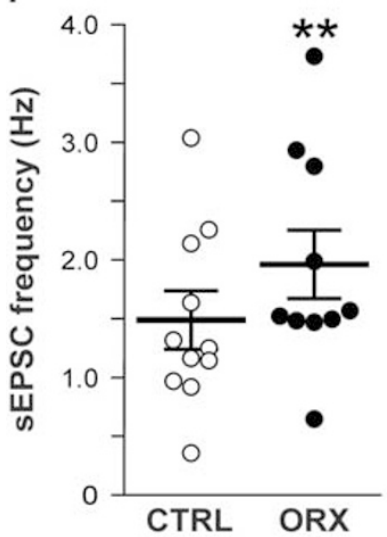

b

CTRL
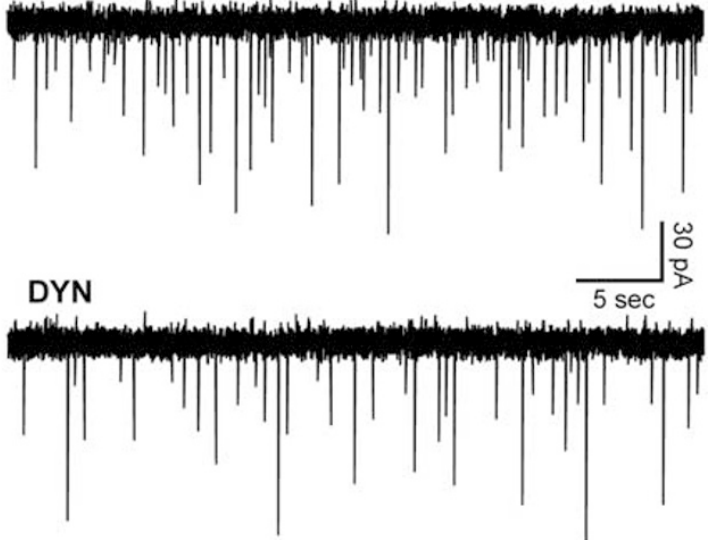

d
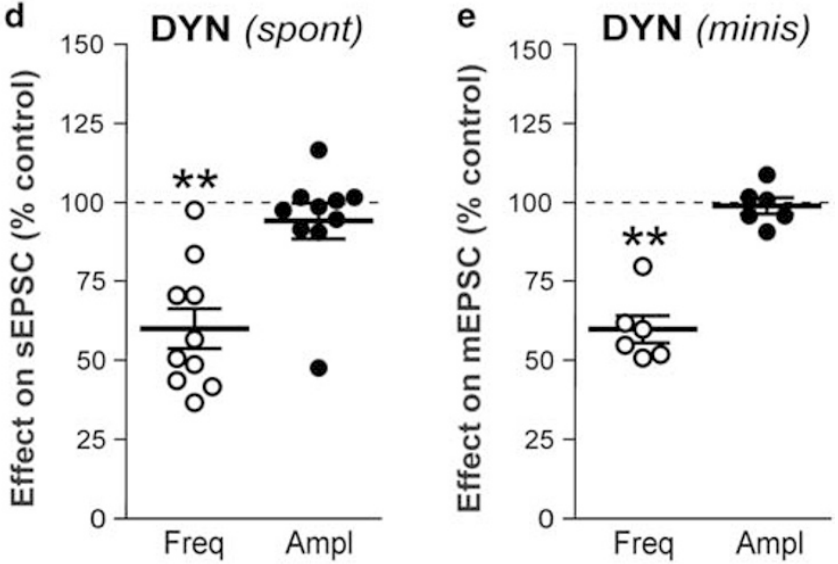

g

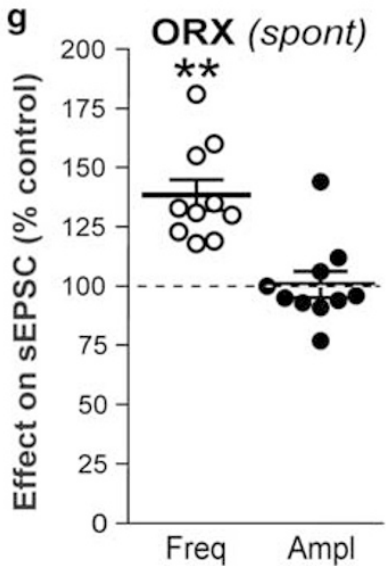

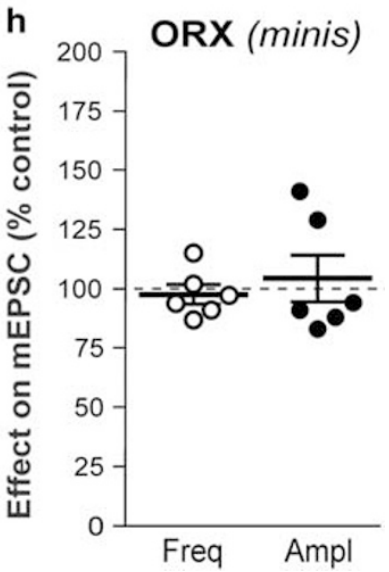

Figure 2 DynA decreased and OrxA increased glutamatergic transmission in pPVT neurons. CTRL, control; DYN, DynA; ORX, OrxA. (a) pPVT slice showing the placement of the recording electrode (infrared optics, $\times 4$ objective). (b) Representative whole-cell current recordings of sEPSCs (downward deflections). The superfusion of $0.2 \mu \mathrm{M}$ DynA decreased the sEPSC frequency by $50 \%$ in this neuron. The resting membrane potential was - $64 \mathrm{mV}$. (c) DynA significantly decreased the mean sEPSC frequency in PVT neurons $(* * 2<0.01$ ). (d) DynA decreased the average sEPSC frequency by $40 \%$, whereas the amplitude was not significantly altered. (e) DynA also decreased the frequency of mEPSCs that were recorded in the presence of tetrodotoxin. ( $f$ ) The superfusion of OrxA significantly increased the sEPSC frequency, an effect that was opposite to DynA. (g) OrxA increased the average sEPSC frequency by $38 \%$, with no effect on amplitude. (h) OrxA had no effect on mEPSCs frequency, in sharp contrast to the effect on sEPSCs.

SCM group (two-way repeated-measures ANOVA; main effect of group (cocaine and SCM), $\mathrm{F}_{1,18}=3.3, p>0.05$; main effect of time (EXT, SHAM, VEH), $\mathrm{F}_{2,36}=0.4, p>0.05$; group $\times$ time interaction, $\mathrm{F}_{2,36}=1.3, p>0.05$; Figure $5 \mathrm{a} 1$ and b1). Responses on the inactive lever did not change during these procedures (Figure 5a1 and b1).

\section{Intra-pPVT Administration of OrxA Induced Cocaine- and SCM-seeking Behavior}

Following the vehicle injections, the mean ( \pm SEM) number of responses was negligible in the two groups $(9.8 \pm 1.4$ for cocaine and $6.2 \pm 0.9$ for SCM; Figure $5 \mathrm{a} 1$ and b1). OrxA 
a

\section{CTRL}

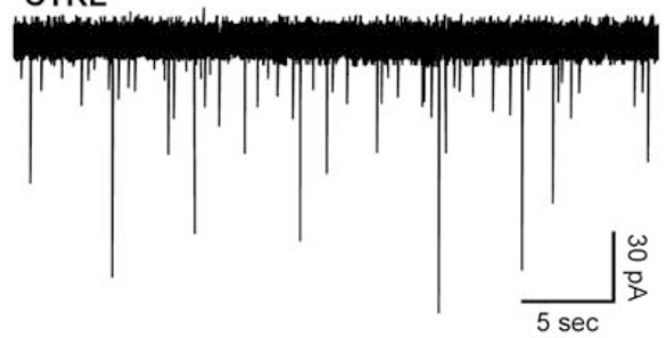

ORX
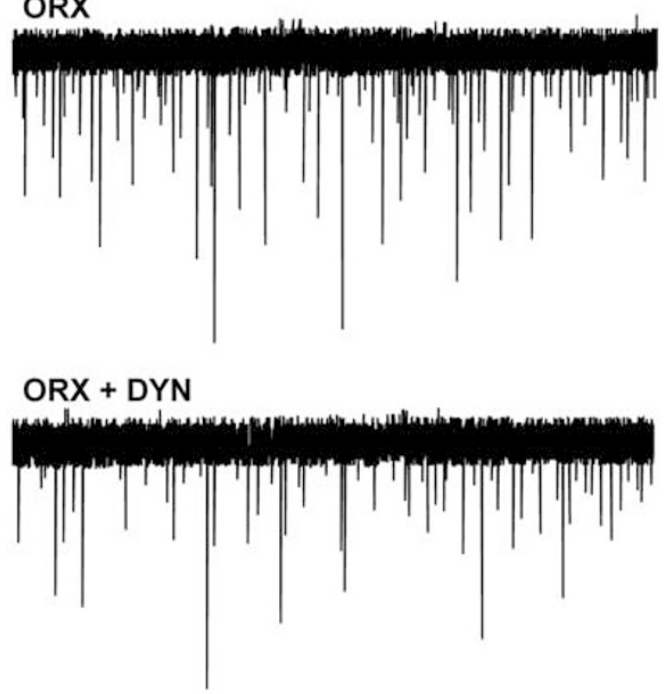

b
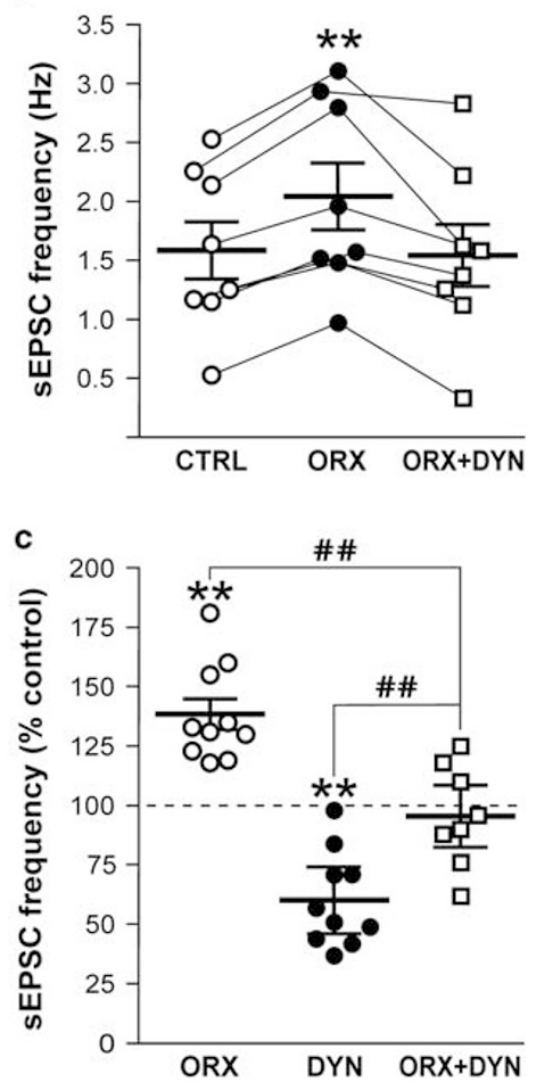

Figure 3 DynA reversed the effect of OrxA on glutamatergic transmission. (a) The superfusion of OrxA ( I $\mu M$ ) increased the sEPSC frequency by $50 \%$. The addition of DynA $(0.2 \mu \mathrm{M})$ in the continued presence of OrxA decreased the sEPSC frequency back to control levels. The resting membrane potential was $-69 \mathrm{mV}$. (b) OrxA applied alone significantly increased the sEPSC frequency, and the addition of DynA together with OrxA reversed this effect (** $p<0.0$ I, vs CTRL). (c) Comparison of sEPSC frequency observed in each experimental group: OrxA alone (38\% increase, from Figure 2e), DynA alone

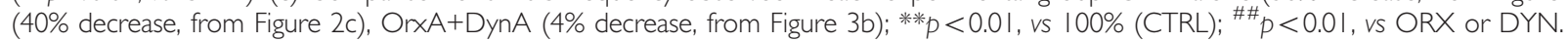

reinstated (primed) cocaine- and SCM-seeking behavior, with a greater effect in the cocaine group (ANOVA followed by Tukey's post hoc test; main effect of group (cocaine and SCM), $F_{1,81}=10.11, p<0.01$; main effect of treatment $(\mathrm{VEH}$, ORX 0.5, DYN 4, ORX 0.5+DYN 1, ORX 0.5+DYN 2, and ORX $0.5+\mathrm{DYN} 4), \mathrm{F}_{5,81}=9.3, p<0.001$; treatment $\times$ group interaction, $\mathrm{F}_{5,81}=3.3, p<0.01$; Figure $5 \mathrm{a} 2$ and $\mathrm{b} 2$ ). Responses on the inactive lever remained low and unaltered for both groups (Figure 5a and b).

\section{Intra-pPVT Administration of DynA Selectively Blocked OrxA-Induced Cocaine-Seeking Behavior}

Cocaine. Separate one-way ANOVAs showed that the coadministration of DynA and OrxA $(n=7 /$ dose) prevented OrxA-induced cocaine-seeking behavior at the highest dose $(4 \mu \mathrm{g})$ tested compared with vehicle $(n=9$; Tukey's post hoc test, $p<0.05$, vs OrxA; ANOVA, $\mathrm{F}_{5,38}=5.8, p<0.001$; Figure $5 \mathrm{~b})$. When administered alone, DynA $(n=7)$ did not produce any effect $(p>0.05$; Figure 5a2). Inactive lever responses were unaffected and identical across treatments (Figure 5a).

Sweetened condensed milk. In contrast to the cocaine group, the co-administration of increasing doses of DynA
( $n=6-8$ animals/dose) with OrxA did not prevent OrxA priming effects compared with vehicle, and OrxA-induced SCM-seeking behavior remained unaffected by DynA (Tukey's post hoc test, $p<0.05$, vs vehicle; ANOVA, $\mathrm{F}_{5,43}=6.5, p<0.001$; Figure 5b2). Inactive lever responses were unaffected (Figure $5 b$ ).

\section{Intra-pPVT Administration of NorBNI Selectively Reversed the Effect of DynA}

To verify that the effect of DynA was KOR specific, a separate group of cocaine LgA rats was prepared. In daily $6 \mathrm{~h}$ cocaine self-administration sessions, the amount $(\mathrm{mg} / \mathrm{kg})$ of cocaine that was self-administered gradually increased (repeatedmeasures ANOVA, $F_{12,240}=13.98, p<0.001$ ) from day 1 $(16.8 \pm 3.0 \mathrm{mg} / \mathrm{kg})$ to day $8 \quad(62.9 \pm 7.1 \mathrm{mg} / \mathrm{kg})$ and then remained unchanged until the end of self-administration training (Figure 4c). By the end of extinction training $(18.8 \pm 0.6$ days), the rats reached a 3 -day average $( \pm$ SEM) number of responses of $7.6 \pm 0.9$. Following NorBNI injections, the mean $( \pm$ SEM) number of responses was negligible and comparable to the extinction and sham responses (one-way ANOVA, $F_{2,33}=0.7646, p=0.4736$; Figure 5c1). One-way ANOVAs showed that NorBNI administration alone did not induce any changes in operant behavior (Figure $5 \mathrm{c} 1$ ). NorBNI 
did not prevent OrxA-induced cocaine-seeking behavior but reversed the effect of DynA (Tukey's post hoc test, $p<0.05$, vs NorBNI; ANOVA, $F_{2,21}=6.326, p=0.0071$; Figure $5 \mathrm{c} 2$ ). Inactive lever responses were unaffected (Figure $5 c$ ).
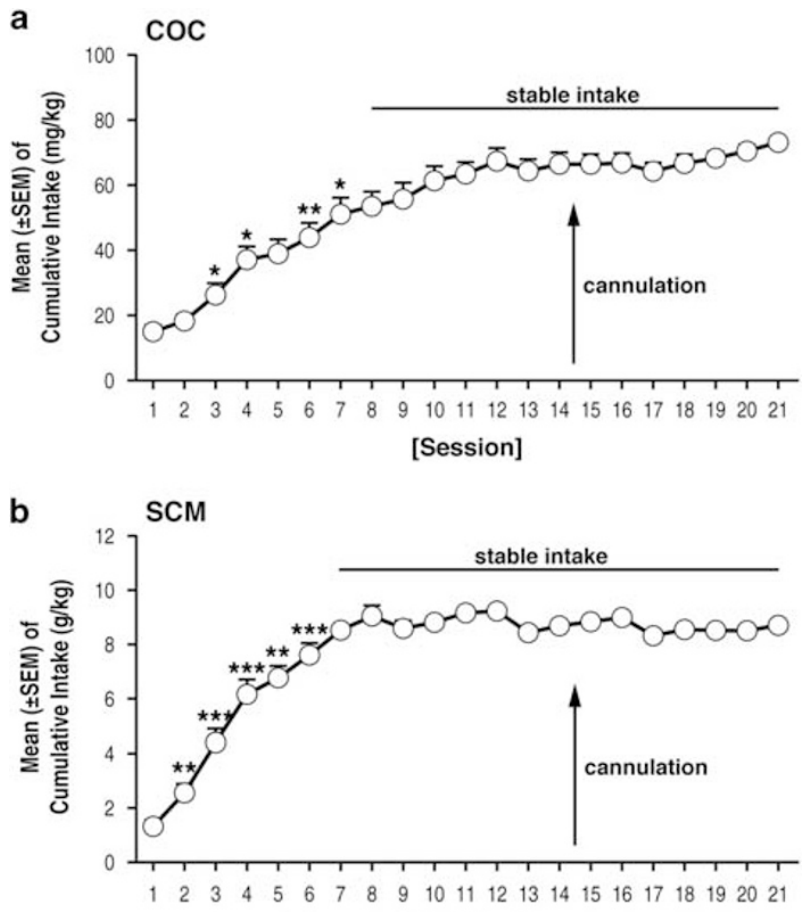

[Session]

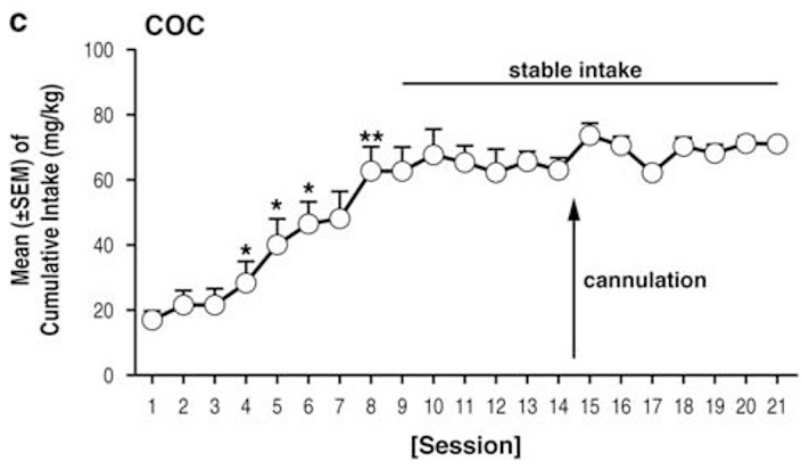

Figure 4 (a-c) Time course of cocaine (COC) ((a) first cohort and (c) second cohort-used to test the effects of KOR blockade on OrxA priming effects) and SCM (b) self-administration over the 21 days of training; $* p<0.05$, $* * * 0.01$, ***** $p<0.00$ I, vs respective previous day (Tukey's post hoc test). The data are expressed as mean \pm SEM; $n=44$ for COC first cohort, $n=12$ for COC second cohort, and $n=49$ for SCM.

\section{DISCUSSION}

Orx and Dyn have opposite effects in the VTA (Baimel et al, 2017; Muschamp et al, 2014). The present study found that such an interaction is also present in the pPVT. The data showed that OrxA increased and DynA decreased glutamatergic transmission in the pPVT. When applied together, these peptides counteracted each other's effects on synaptic activity. Although an earlier study demonstrated the importance of Orx transmission in the PVT during cocaine-seeking behavior (Matzeu et al, 2016), the present study found that discrete administration of OrxA in the pPVT reinstated extinguished both SCM-seeking behavior and cocaine-seeking behavior, and the magnitude of cocaine seeking was significantly higher vs SCM. Furthermore, this effect was prevented by DynA only in animals that had a history of extended access to cocaine and was reversed by NorBNI, confirming that the DynA-induced blockade of the effects of OrxA occurred through actions on KORs. As suggested by others (Muschamp et al, 2014), these results support the hypothesis that targeting KORs may have beneficial effects for the treatment of disorders that are associated with increased Orx activity.

Electrophysiological investigations have shown that Orx enhances neuronal excitability via pre- and postsynaptic mechanisms (Kukkonen, 2013; Leonard and Kukkonen, 2014). Consistent with the dense projections of Orx neurons to the PVT and mostly glutamatergic nature of the PVT network, we observed an increase in Orx-induced glutamate release in pPVT neurons that was comparable to the effects that were reported in the hypothalamus ( $\mathrm{Li}$ et al, 2002; van den Pol et al, 1998), laterodorsal tegmental area (Burlet et al, 2002), nucleus tractus solitarius (Smith et al, 2002), and neocortex (Aracri et al, 2015). Previous studies that evaluated the PVT, however, found no effect of Orx on the frequency of sEPSCs in transgenic mice (Huang et al, 2006) or evoked excitatory transmission in rats (Kolaj et al, 2007). The use of a different species (Huang et al, 2006) or different experimental designs (Kolaj et al, 2007) may account for such discrepancies. The decrease in glutamatergic transmission that we observed upon DynA application was similar to the effects that were reported in other brain regions (Crowley et al, 2016; Wagner et al, 1993). In the present study, OrxA and DynA modulated glutamate signaling in opposite directions, and DynA completely reversed the effects of OrxA, suggesting that these two systems balance each other to regulate neuronal activity in the PVT. Orexin and Dyn also have opposing actions on neuronal firing rate in the VTA and counteract each other upon co-release (Muschamp et al, 2014). Similarly, Dyn has been shown to inhibit the increase in firing rate that is elicited by Orx in hypothalamic

Figure 5 (al, b l, cl) Cocaine (COC) and SCM animals presented comparable responses at the end of extinction, following sham and vehicle (or NorBNI $(c I)$ ) injections. EXT, extinction; SHAM, sham injection; VEH, vehicle; DYN, dynorphin-A; ORX, orexin-A; NorBNI, nor-binaltorphimine dihydrochloride. (a2) Intra-pPVT administration of OrxA but not DynA induced cocaine-seeking behavior; *** $p<0.0$ I, vs VEH. DynA (I and $2 \mu g$ ) did not affect OrxA primeinduced reinstatement; ${ }^{*} p<0.05$, $* * * *<0.00 \mathrm{I}$, vs $\vee \mathrm{VH}$. DynA $(4 \mu \mathrm{g})$ reversed OrxA prime-induced reinstatement; ${ }^{*} p<0.0 \mathrm{I}$, vs ORX. The data are expressed as mean \pm SEM; $n=9-7$ animals/group. (b2) Intra-pPVT administration of OrxA but not DynA induced SCM-seeking behavior. DynA did not affect OrxA prime-induced reinstatement; $* p<0.05$, *** $p<0.0$ I, **** $p<0.00$ I, vs VEH. The data are expressed as mean \pm SEM; $n=6-1$ I animals/group. (a2, b2) Greater effects of OrxA in cocaine vs SCM animals; ${ }^{+} p<0.05$, vs respective treatment in cocaine group. (c2) NorBNI did not reinstate or potentiate cocaineseeking behavior induced by OrxA alone, but it reversed the inhibitory action of DynA; ${ }^{*} p<0.05$ vs NorBNI. The data are expressed as mean \pm SEM; $n=5-7$ animals/group. 

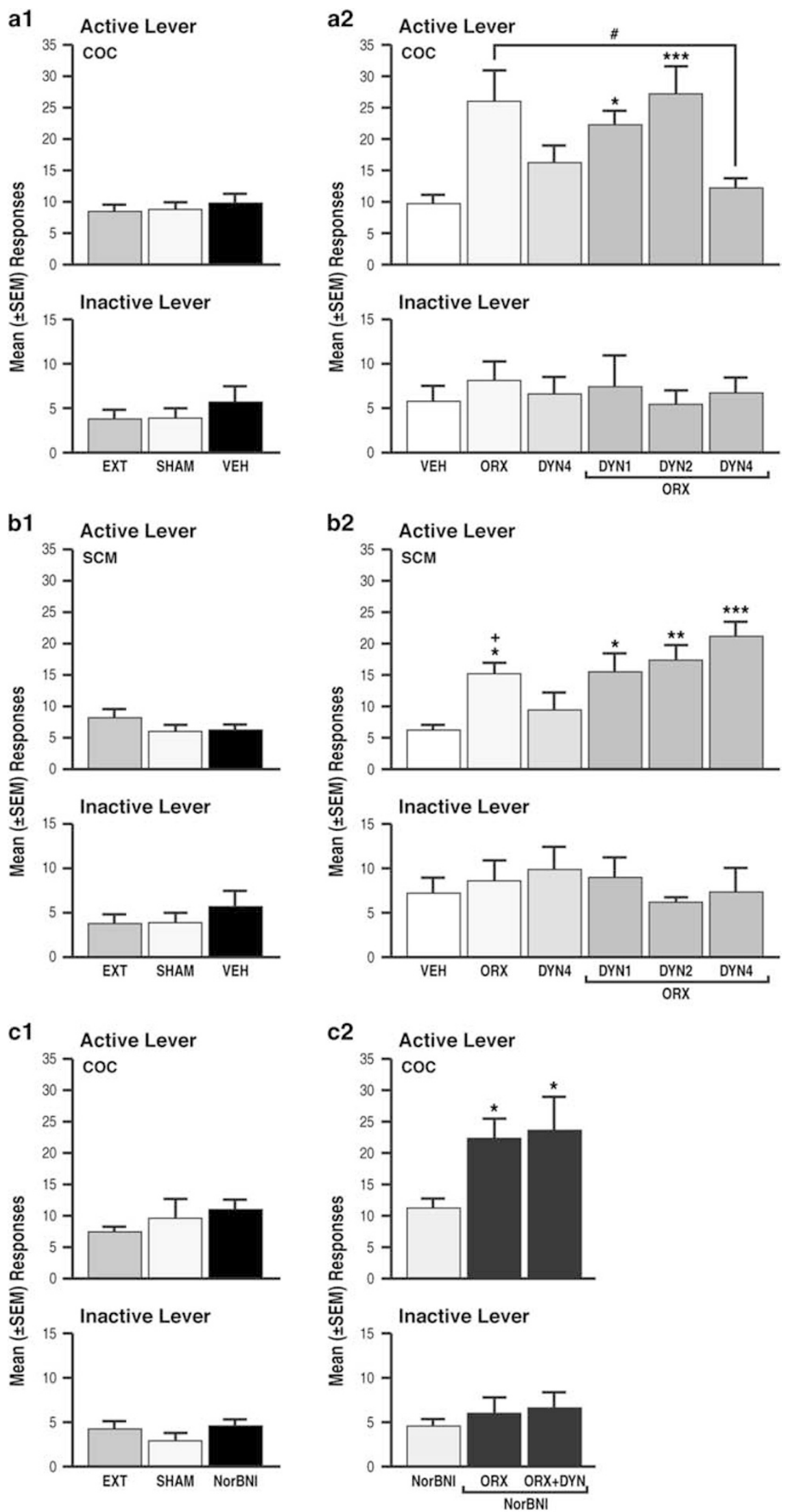
neurons (Li and van den Pol, 2006) and counterbalance the response to Orx in basal forebrain neurons, preventing overexcitation (Ferrari et al, 2016). Importantly, our cellular findings are consistent with the behavioral data, indicating a functional interaction between the Orx and Dyn systems.

The recording of spontaneous and miniature synaptic events, respectively, provided information on action potentialdependent (network activity) and -independent (vesicular release) glutamate transmission in the pPVT. DynA decreased both sEPSCs and mEPSCs, indicating that the modulation of baseline excitatory signaling by DynA takes place on a site located at or near the presynaptic terminal. OrxA, however, increased the frequency of sEPSCs but had no effect on mEPSCs, indicating that action-dependent transmitter release and thus network activity are required to drive the effects of OrxA on baseline glutamatergic activity in the PVT. In addition, DynA and OrxA affected only the frequency and not amplitude of the recorded synaptic events, indicating that the effect of both peptides is presynaptic and solely target glutamate release, with no involvement of postsynaptic efficacy. The Orx system appears to regulate glutamate release in the PVT by modulating a synaptic network, whereas DynA acts locally on presynaptic terminals. Thus, DynA and OrxA may operate at different loci in the pPVT, perhaps implicating different circuits as recently proposed in the VTA where the sensitivity to OrxA was circuit specific (Baimel et al, 2017). The nature of the network that is targeted by OrxA in the PVT remains to be determined.

The observation that local injections of OrxA in the pPVT induced cocaine- and SCM-seeking behavior strongly supports the hypothesis that Orx projections to the pPVT are important in the modulation of reward-seeking behavior in general. An explanation for the general priming effects of OrxA for both cocaine and SCM could involve the known involvement of the Orx system in arousal. Orexin is well known to regulate general arousal (Sakurai et al, 2010), and the anticipation of food reward activates Orx-containing neurons in the PVT (Choi et al, 2010). The medial prefrontal cortex is one target of Orx-activated PVT neurons (Huang et al, 2006; Ishibashi et al, 2005). The present results suggest that Orx inputs to the PVT facilitate cortical activation that is linked to general arousal (Sato-Suzuki et al, 2002) that could explain the nonspecific effect of intra-pPVT OrxA injections on both cocaine and SCM reinstatement.

Although hypothalamic Orx neurons represent the exclusive source of OrxA to the pPVT (Li and Kirouac, 2012; Peyron et al, 1998), DynA is expressed in other brain regions that are connected to the pPVT, such as the prefrontal cortex and central amygdala (Healy and Meador-Woodruff, 1994; Li and Kirouac, 2012; Ramsdell and Meador-Woodruff, 1993; Watson et al, 1982; Weber et al, 1982), that indeed could modulate OrxA transmission in the pPVT. For example, the prefrontal cortex is well known to be involved in the executive control over drug seeking (Kalivas et al, 2005a; Kalivas and Volkow, 2005b). The prefrontal cortex has been shown to project to the pPVT (Li and Kirouac, 2012) and presumably supplies a considerable Dyn input and thus may modulate the effects of Orx in the pPVT. However, to our knowledge, the modulation of cocaine-seeking behavior by Dyn that derives from other sources than hypothalamic Orx neurons to the pPVT has not yet been characterized and needs to be further investigated.

OrxA produced greater reinstatement in the cocaine group vs SCM group, and DynA prevented OrxA-induced cocaine-seeking behavior but had no effect on SCM seeking when injected alone. One possible explanation for the differential effects of OrxA and DynA on cocaine- and SCM-seeking behavior is that cocaine-induced neuroadaptations amplified the motivational effects of OrxA-pPVT signaling following abstinence. The greater OrxA priming effect on cocaine-seeking behavior may also partially explain the greater efficacy of DynA in the cocaine group compared with the SCM group. Orexin and Dyn are colocalized and coreleased (Chou et al, 2001; Li and van den Pol, 2006), and one may speculate that after long access to cocaine, both the Orx and Dyn systems are sensitized, reflected by a greater priming effect of OrxA and greater inhibitory effect of DynA. Dysregulation of the Orx and Dyn systems by cocaine has been reported previously. For example, the Orx system was engaged to a greater extent by drugs of abuse than by SCM in an operant model of reward seeking (ie, conditioned reinstatement; Martin-Fardon et al, 2016; Martin-Fardon and Weiss, 2014; Matzeu et al, 2016). These results are consistent with the hypothesis that cocaine produces neuroadaptations in the circuits that control the motivation for natural rewards. Cocaine-induced neuroadaptations of the Dyn system have been well documented. Cocaine enhances Dyn expression, KOR signaling, and KOR levels in the striatum and VTA (Daunais et al, 1993; Piras et al, 2010; Spangler et al, 1996; Unterwald et al, 1994) and upregulates $P d y n$ gene expression in the nucleus accumbens (Caputi et al, 2014). KOR downregulation was found in the basolateral amygdala and septum in animals that were withdrawn from binge cocaine for 14 days (Bailey et al, 2007). In the present study, NorBNI did not reinstate cocaine-seeking behavior and did not potentiate cocaineseeking behavior that was induced by OrxA alone, but it reversed the inhibitory actions of DynA (Figure 5c2), implicating KORs in the effects of DynA. These data are difficult to reconcile with the findings that DynA reversed the effect of OrxA (Figure 5a2). Intuitively, antagonizing KOR should have enhanced OrxA priming effects. However, the observation that KOR blockade did not exacerbate OrxA priming effects suggests that endogenous DynA does not play a role in OrxA-induced reinstatement of cocaine-seeking behavior. This will need to be further investigated.

Overall, the present study demonstrates that DynA and OrxA have opposite actions on glutamatergic transmission to balance neuronal activity in the pPVT and control cocaineseeking behavior. These results suggest that DynA in the pPVT may limit cocaine seeking at least partially by counteracting the OrxA-dependent increase in glutamate release and suggest that concomitant intervention at both Orx/OrxR and Dyn/KOR systems may represent valuable pharmacological targets to prevent cocaine relapse.

\section{FUNDING AND DISCLOSURE}

The authors declare no conflict of interest.

\section{ACKNOWLEDGMENTS}

This is publication number 29472 from The Scripps Research Institute. We thank Michael Arends for assistance with 
manuscript preparation. This work was supported by the National Institute on Drug Abuse and National Institute on Alcohol Abuse and Alcoholism (grant nos. DA033344, AA024146, AA006420, and AA022249 to RM-F and AA020608, AA006420, AA026081, and DA043799 to OG).

\section{REFERENCES}

Ammoun S, Holmqvist T, Shariatmadari R, Oonk HB, Detheux M, Parmentier $M$ et al (2003). Distinct recognition of OX1 and OX2 receptors by orexin peptides. J Pharmacol Exp Ther 305: 507-514.

Aracri P, Banfi D, Pasini ME, Amadeo A, Becchetti A (2015). Hypocretin (orexin) regulates glutamate input to fast-spiking interneurons in layer $\mathrm{V}$ of the Fr2 region of the murine prefrontal cortex. Cereb Cortex 25: 1330-1347.

Arcelli P, Frassoni C, Regondi MC, De Biasi S, Spreafico R (1997). GABAergic neurons in mammalian thalamus: a marker of thalamic complexity? Brain Res Bull 42: 27-37.

Bailey A, Gianotti R, Ho A, Kreek MJ (2007). Downregulation of kappa-opioid receptors in basolateral amygdala and septum of rats withdrawn for 14 days from an escalating dose "binge" cocaine administration paradigm. Synapse 61: 820-826.

Baimel C, Lau BK, Qiao M, Borgland SL (2017). Projection-targetdefined effects of orexin and dynorphin on VTA dopamine neurons. Cell Rep 18: 1346-1355.

Bruchas MR, Land BB, Chavkin C (2010). The dynorphin/kappa opioid system as a modulator of stress-induced and pro-addictive behaviors. Brain Res 1314: 44-55.

Burlet S, Tyler CJ, Leonard CS (2002). Direct and indirect excitation of laterodorsal tegmental neurons by Hypocretin/Orexin peptides: implications for wakefulness and narcolepsy. J Neurosci 22: 2862-2872.

Caputi FF, Carretta D, Tzschentke TM, Candeletti S, Romualdi P (2014). Opioid receptor gene expression in human neuroblastoma SH-SY5Y cells following tapentadol exposure. J Mol Neurosci 53: 669-676.

Chavkin C, James IF, Goldstein A (1982). Dynorphin is a specific endogenous ligand of the kappa opioid receptor. Science 215: 413-415.

Chen Z, Tang Y, Tao H, Li C, Zhang X, Liu Y (2015). Dynorphin activation of kappa opioid receptor reduces neuronal excitability in the paraventricular nucleus of mouse thalamus. Neuropharmacology 97: 259-269.

Choi DL, Davis JF, Fitzgerald ME, Benoit SC (2010). The role of orexin-A in food motivation, reward-based feeding behavior and food-induced neuronal activation in rats. Neuroscience 167: $11-20$.

Chou TC, Lee CE, Lu J, Elmquist JK, Hara J, Willie JT et al (2001). Orexin (hypocretin) neurons contain dynorphin. J Neurosci 21: RC168.

Crowley NA, Bloodgood DW, Hardaway JA, Kendra AM, McCall JG, Al-Hasani R et al (2016). Dynorphin controls the gain of an amygdalar anxiety circuit. Cell Rep 14: 2774-2783.

Daunais JB, Roberts DC, McGinty JF (1993). Cocaine selfadministration increases preprodynorphin, but not c-fos, mRNA in rat striatum. Neuroreport 4: 543-546.

deLecea L, Kilduff TS, Peyron C, Gao X, Foye PE, Danielson PE et al (1998). The hypocretins: hypothalamus-specific peptides with neuroexcitatory activity. Proc Natl Acad Sci USA 95: 322-327.

Ferrari LL, Agostinelli LJ, Krashes MJ, Lowell BB, Scammell TE, Arrigoni E (2016). Dynorphin inhibits basal forebrain cholinergic neurons by pre- and postsynaptic mechanisms. J Physiol 594: 1069-1085.

Harris GC, Wimmer M, Aston-Jones G (2005). A role for lateral hypothalamic orexin neurons in reward seeking. Nature 437: 556-559.
Healy DJ, Meador-Woodruff JH (1994). Prodynorphin-derived peptide expression in primate cortex and striatum. Neuropeptides 27: 277-284.

Huang H, Ghosh P, van den Pol AN (2006). Prefrontal cortexprojecting glutamatergic thalamic paraventricular nucleus-excited by hypocretin: a feedforward circuit that may enhance cognitive arousal. J Neurophysiol 95: 1656-1668.

Ishibashi M, Takano S, Yanagida H, Takatsuna M, Nakajima K, Oomura Y et al (2005). Effects of orexins/hypocretins on neuronal activity in the paraventricular nucleus of the thalamus in rats in vitro. Peptides 26: 471-481.

James MH, Mahler SV, Moorman DE, Aston-Jones G (2016). A decade of orexin/hypocretin and addiction: where are we now? Curr Top Behav Neurosci 33: 247-281.

Kalivas PW, Volkow N, Seamans J (2005a). Unmanageable motivation in addiction: a pathology in prefrontal-accumbens glutamate transmission. Neuron 45: 647-650.

Kalivas PW, Volkow ND (2005b). The neural basis of addiction: a pathology of motivation and choice. Am J Psychiatry 162: 1403-1413.

Kirouac GJ, Parsons MP, Li S (2005). Orexin (hypocretin) innervation of the paraventricular nucleus of the thalamus. Brain Res 1059: 179-188.

Kolaj M, Doroshenko P, Yan Cao X, Coderre E, Renaud LP (2007). Orexin-induced modulation of state-dependent intrinsic properties in thalamic paraventricular nucleus neurons attenuates action potential patterning and frequency. Neuroscience 147: 1066-1075.

Kolaj M, Zhang L, Hermes ML, Renaud LP (2014). Intrinsic properties and neuropharmacology of midline paraventricular thalamic nucleus neurons. Front Behav Neurosci 8: 132.

Kukkonen JP (2013). Physiology of the orexinergic/hypocretinergic system: a revisit in 2012. Am J Physiol Cell Physiol 304: C2-32.

Leonard CS, Kukkonen JP (2014). Orexin/hypocretin receptor signalling: a functional perspective. Br J Pharmacol 171: 294-313.

Li S, Kirouac GJ (2012). Sources of inputs to the anterior and posterior aspects of the paraventricular nucleus of the thalamus. Brain Struct Funct 217: 257-273.

Li SB, Giardino WJ, de Lecea L (2016). Hypocretins and arousal. Curr Top Behav Neurosci 33: 93-104.

Li Y, Gao XB, Sakurai T, van den Pol AN (2002). Hypocretin/ Orexin excites hypocretin neurons via a local glutamate neuron-A potential mechanism for orchestrating the hypothalamic arousal system. Neuron 36: 1169-1181.

Li Y, van den Pol AN (2006). Differential target-dependent actions of coexpressed inhibitory dynorphin and excitatory hypocretin/ orexin neuropeptides. J Neurosci 26: 13037-13047.

Mansour A, Burke S, Pavlic RJ, Akil H, Watson SJ (1996). Immunohistochemical localization of the cloned kappa 1 receptor in the rat CNS and pituitary. Neuroscience 71: 671-690.

Martin-Fardon R, Cauvi G, Kerr TM, Weiss F (2016). Differential role of hypothalamic orexin/hypocretin neurons in reward seeking motivated by cocaine versus palatable food. Addict Biol (epub ahead of print).

Martin-Fardon R, Weiss F (2014). Blockade of hypocretin receptor1 preferentially prevents cocaine seeking: comparison with natural reward seeking. Neuroreport 25: 485-488.

Matzeu A, Cauvi G, Kerr TM, Weiss F, Martin-Fardon R (2017). The paraventricular nucleus of the thalamus is differentially recruited by stimuli conditioned to the availability of cocaine versus palatable food. Addict Biol 22: 70-77.

Matzeu A, Kerr TM, Weiss F, Martin-Fardon R (2016). Orexin-A/ hypocretin-1 mediates cocaine-seeking behavior in the posterior paraventricular nucleus of the thalamus via orexin/hypocretin receptor-2. J Pharmacol Exp Ther 359: 273-279.

Matzeu A, Weiss F, Martin-Fardon R (2015). Transient inactivation of the posterior paraventricular nucleus of the thalamus blocks cocaine-seeking behavior. Neurosci Lett 608: 34-39.

Matzeu A, Zamora-Martinez ER, Martin-Fardon R (2014). The paraventricular nucleus of the thalamus is recruited by both 
natural rewards and drugs of abuse: recent evidence of a pivotal role for orexin/hypocretin signaling in this thalamic nucleus in drug-seeking behavior. Front Behav Neurosci 8: 117.

Muschamp JW, Hollander JA, Thompson JL, Voren G, Hassinger LC, Onvani S et al (2014). Hypocretin (orexin) facilitates reward by attenuating the antireward effects of its cotransmitter dynorphin in ventral tegmental area. Proc Natl Acad Sci USA 111: E1648-E1655.

Paxinos G, Watson C (1997). The Rat Brain in Stereotaxic Coordinates2nd ednAcademic Press: San Diego.

Peyron C, Tighe DK, van den Pol AN, de Lecea L, Heller HC, Sutcliffe JG et al (1998). Neurons containing hypocretin (orexin) project to multiple neuronal systems. J Neurosci 18: 9996-10015.

Piras AP, Zhou Y, Schlussman SD, Ho A, Kreek MJ (2010). Acute withdrawal from chronic escalating-dose binge cocaine administration alters kappa opioid receptor stimulation of [35S] guanosine 5'-O-[gamma-thio]triphosphate acid binding in the rat ventral tegmental area. Neuroscience 169: 751-757.

Ramsdell CD, Meador-Woodruff JH (1993). Expression of prodynorphin-derived peptides and mRNA in guineapig cortex. Neuropeptides 25: 131-138.

Sakurai T, Amemiya A, Ishii M, Matsuzaki I, Chemelli RM, Tanaka $\mathrm{H}$ et al (1998). Orexins and orexin receptors: a family of hypothalamic neuropeptides and $\mathrm{G}$ protein-coupled receptors that regulate feeding behavior. Cell 92: 573-585.

Sakurai T, Mieda M, Tsujino N (2010). The orexin system: roles in sleep/wake regulation. Ann NY Acad Sci 1200: 149-161.

Sato-Suzuki I, Kita I, Seki Y, Oguri M, Arita H (2002). Cortical arousal induced by microinjection of orexins into the paraventricular nucleus of the rat. Behav Brain Res 128: 169-177.

Schwarzer C (2009). 30 years of dynorphins-new insights on their functions in neuropsychiatric diseases. Pharmacol Ther 123: 353-370.

Smith BN, Davis SF, Van Den Pol AN, Xu W (2002). Selective enhancement of excitatory synaptic activity in the rat nucleus tractus solitarius by hypocretin 2. Neuroscience 115: 707-714.
Spangler R, Ho A, Zhou Y, Maggos CE, Yuferov V, Kreek MJ (1996). Regulation of kappa opioid receptor mRNA in the rat brain by "binge' pattern cocaine administration and correlation with preprodynorphin mRNA. Brain Res Mol Brain Res 38: 71-76.

Tsujino N, Sakurai T (2013). Role of orexin in modulating arousal, feeding, and motivation. Front Behav Neurosci 7: 28.

Unterwald EM, Rubenfeld JM, Kreek MJ (1994). Repeated cocaine administration upregulates kappa and mu, but not delta, opioid receptors. Neuroreport 5: 1613-1616.

van den Pol AN, Gao XB, Obrietan K, Kilduff TS, Belousov AB (1998). Presynaptic and postsynaptic actions and modulation of neuroendocrine neurons by a new hypothalamic peptide, hypocretin/orexin. J Neurosci 18: 7962-7971.

Van der Werf YD, Witter MP, Groenewegen HJ (2002). The intralaminar and midline nuclei of the thalamus. Anatomical and functional evidence for participation in processes of arousal and awareness. Brain Res Brain Res Rev 39: 107-140.

Wagner JJ, Terman GW, Chavkin C (1993). Endogenous dynorphins inhibit excitatory neurotransmission and block LTP induction in the hippocampus. Nature 363: 451-454.

Watson SJ, Khachaturian H, Akil H, Coy DH, Goldstein A (1982). Comparison of the distribution of dynorphin systems and enkephalin systems in brain. Science 218: 1134-1136.

Weber E, Evans CJ, Barchas JD (1982). Predominance of the aminoterminal octapeptide fragment of dynorphin in rat brain regions. Nature 299: 77-79.

Wee S, Koob GF (2010). The role of the dynorphin-kappa opioid system in the reinforcing effects of drugs of abuse. Psychopharmacology 210: 121-135.

Whitfield TW Jr, Schlosburg JE, Wee S, Gould A, George O, Grant $Y$ et al (2015). kappa Opioid receptors in the nucleus accumbens shell mediate escalation of methamphetamine intake. The $J$ Neurosci 35: 4296-4305. 